\title{
A modified pseudo-rigid-body modeling approach for compliant mechanisms with fixed-guided beam flexures
}

\author{
Pengbo Liu ${ }^{1}$ and Peng Yan ${ }^{1,2}$ \\ ${ }^{1}$ Key Laboratory of High-efficiency and Clean Mechanical Manufacture, Ministry of Education, School of \\ Mechanical Engineering, Shandong University, Jinan, Shandong, 250061, China \\ ${ }^{2}$ School of Automation Science and Electrical Engineering, Beihang University, Beijing, 100191, China
}

Correspondence: Peng Yan (pengyan2007@gmail.com)

Received: 2 August 2017 - Accepted: 9 November 2017 - Published: 12 December 2017

\begin{abstract}
In the present paper, we investigate a modified pseudo-rigid-body (MPRB) modeling approach for compliant mechanisms with fixed-guided beam flexures by considering the nonlinear effects of center-shifting and load-stiffening. In particular, a fixed-guided compliant beam is modeled as a pair of fixed-free compliant beams jointed at the inflection point, where each fixed-free beam flexure is further modeled by a rigid link connected with an extension spring by a torsion spring, based on the beam constraint model (BCM). Meanwhile, the characteristic parameters of the proposed MPRB model are no longer constant values, but affected by the applied general tip load, especially the axial force. The developed MPRB modeling method is then applied to the analysis of three common compliant mechanisms (i.e. compound parallelogram mechanisms, bistable mechanisms and 1-DOF translational mechanisms), which is further verified by the finite element analysis (FEA) results. The proposed MPRB model provides a more accurate method to predict the performance characteristics such as deformation capability, stiffness variation, as well as error motions of complaint mechanisms with fixed-guided beam flexures, and offers a new look into the design and optimization of beam-based compliant mechanisms.
\end{abstract}

\section{Introduction}

Compliant mechanisms are flexible structures that transmit motions or forces through elastic deformations with the advantages of low-cost and high-performance by effectively eliminating the impacts of frictions, wears and backlashes (Howell, 2001; Turkkan and Su, 2016). Thanks to the capability to deliver high precision motions, compliant mechanisms have been widely explored in advanced applications of precision engineering including micro/nanomanipulating systems, biological cell manipulations and precision instruments (Liu et al., 2015; Ding et al., 2017). Meanwhile significant research efforts have been devoted to the design and analysis of compliant mechanisms (Liu and Yan, 2015; Hao, 2016).

In particular, the fixed-guided beam flexure is a representative type of flexible segments in compliant mechanisms receiving increasing attentions in research literatures due to their potential to achieve large translational motions such as bistable mechanisms (Chen and Ma, 2015), compliant parallelogram mechanisms (Luo and Liu, 2014), and compound compliant parallelogram mechanisms (Hao and $\mathrm{Li}, 2016$ ). With one end remaining a constant angle, the deflected configuration of the fixed-guided beam carries at least one inflection point, where axial deflections and axial forces have significant impacts on its mechanical properties. In particular, the equivalent stiffness will change and the rotational center will shift with respect to the connected links in the presence of load applications and the subsequent deflections. Consequently, complaint mechanisms with fixed-guided beams inevitably suffer from performance tradeoffs in terms of travel range, static stiffness and motion precision, see $\mathrm{Hao}$ and $\mathrm{Li}$ (2016), Teo et al. (2010), Ma and Chen (2017) and the references therein.

Various methods have been developed for the analysis and design of complaint mechanisms with beam flexures, such as the finite element analysis, elliptical integrals, beam constraint model, as well as topological synthesis. Consid- 
ering the complicated calculation of these approach, Howell (2001) proposed the pseudo-rigid-body (PRB) modeling method to provide a simplified approach to analyze the deflection of beam flexures, which consists of two rigid links joined at a pin joint with a torsion spring. Based on the concept of PRB, Midha et al. (2015) presented a viable method of analyzing a fixed-guided compliant beam with an inflection point for various boundary conditions. To further approximate tip deflection of cantilever beams subject to combined end forces and moments, the PRB 2R (revolute) (Yu et al., 2012) and 3R (Su, 2009) have been explored to improve the analysis accuracy. However these PRB models can only describe bending deformation, without capturing the axial deformation of the beams. To this end, the PRB PR (prismatic-revolute) (Yu et al., 2015), PRR (Yu et al., 2016) and 3-Spring model (Venkiteswaran and $\mathrm{Su}, 2016$ ) have been developed by adopting a prismatic pair with a linear spring to describe the axial deformations. Note that the PRB parameters are usually optimized over a large range of deflections. These models demonstrate significant modeling error for the small deflection beams adopted in the nano-manipulating systems, especially when the nonlinear effect caused by axial forces and deformations is addressed.

In this paper, we focus on the pseudo-rigid-body modeling of the fixed-guided beams employed in the nanomanipulating systems, where the transverse displacements is an order of magnitude less than the beam length but generally greater than the beam nominal thickness. Based on the beam constraint model (BCM) (Awtar et al., 2007), a modified pseudo-rigid-body (MPRB) modeling approach is proposed for the fixed-guided beam flexures by taking the impacts of nonlinear center-shifting and load-stiffening into account, where extension springs representing the axial stiffness and torsion springs accounting for the bending stiffness are adopted. Different from the existing PRB models, the PRB parameters of the proposed MPRB model are determined by the general tip loads. The model is successfully applied to the analysis of the compound parallelogram mechanism, the bistable mechanism and the 1-DOF translational mechanism, where the analytical results and FEA results demonstrate the effectiveness and accuracy of the proposed MPRB method.

In the rest of the paper, the BCM is first recalled in Sect. 2. In Sect. 3, the MPRB modeling method for a fixed-free beam flexure is developed. Section 4 establishes the MPRB model for the fixed-guided beam flexure, where the FEA analysis is also deployed to verify the effectiveness of the proposed method. In Sect. 5 the proposed MPRB modeling approach is applied to three common compliant mechanisms as case studies, where comparisons with existing methods are also provided. Finally, some concluding remarks are summarized in Sect. 6.

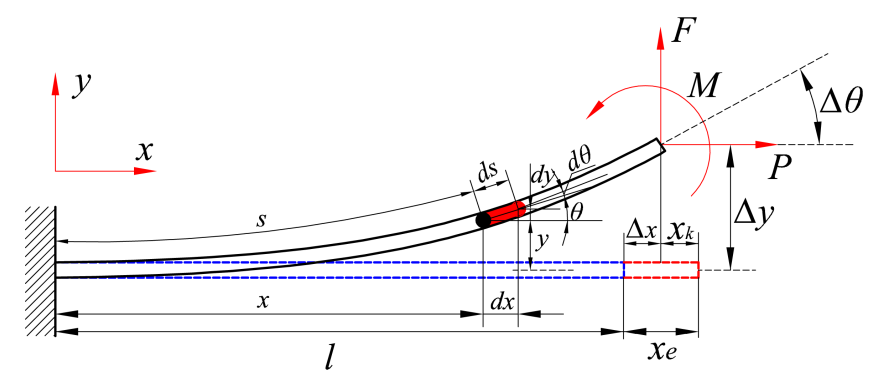

Figure 1. Deflection of a cantilever beam subject to a combined end force and moment.

\section{Beam constraint model}

We start with a fixed-free beam with generalized end forces as depicted in Fig. 1. Note that we would like to focus on the deformed configuration that the transverse displacements are an order of magnitude less than the beam length but generally greater than the beam thickness, which agrees with many actual applications of nano-manipulating systems (Parmar et al., 2014). Therefore, the beam curvature can be linearized by assuming small slopes. Based on the Euler-Bernoulli equation, we have

$M(x)=E I \frac{\mathrm{d} \theta}{\mathrm{d} s}=E I \frac{\mathrm{d}^{2} y}{\mathrm{~d} x^{2}}$,
$M(x)=M+F\left(l+\Delta_{x}-x\right)-P\left(\Delta_{y}-y\right)$,

where $M(x)$ is the equivalent bending moment applied at the arbitrary cross section, $F, P$ and $M$ are the applied transverse force, axial force and bending moment, $x$ is the distance along the undeflected beam axis, $y$ is the transverse deflection, $\theta$ is the angular deflection, $\mathrm{d} \theta / \mathrm{d} s$ is the change rate of the angular deflection along the beam, $E$ is the Young's modulus, $I$ is the inertia moment, $l$ is the initial length of the beam, $\Delta_{x}$ and $\Delta_{y}$ are the axial and transverse deformations of the tip point, respectively.

The above equations can be solved by applying the boundary conditions that $y=0, \frac{\mathrm{d} y}{\mathrm{~d} x}=0$ at $x=0$ and $\frac{\mathrm{d}^{2} y}{\mathrm{~d} x^{2}}=M / E I$, $\frac{\mathrm{d}^{3} y}{\mathrm{~d} x^{3}}=(P \mathrm{~d} y / \mathrm{d} x-F) / E I$ at $x=l+\Delta_{x}$. The results can be further approximated by recalling Awtar et al. (2007) as

$$
\begin{aligned}
\delta_{y}= & \frac{\Delta_{y}}{l} \approx \frac{5 f}{3(5+2 p)}+\frac{12 m}{2(12+5 p)}, \\
\delta_{\theta} \approx & \frac{12 f}{2(12+5 p)}+\frac{48(10+p) m}{5 p^{2}+208 p+480}, \\
\delta_{x}= & \frac{\Delta_{x}}{l}=\frac{x_{\mathrm{e}}}{l}-\frac{x_{\mathrm{k}}}{l}, \\
\delta_{x \mathrm{e}}= & \frac{x_{\mathrm{e}}}{l}=\frac{t^{2} p}{12}, \\
\delta_{x \mathrm{k}}= & \frac{x_{\mathrm{k}}}{l} \approx \frac{420-p}{700} \delta_{y}^{2}-\frac{70-p}{700} \delta_{y} \delta_{\theta} \\
& +\frac{420-11 p}{6300} \delta_{\theta}^{2},
\end{aligned}
$$


where $f=\frac{F l^{2}}{E I}, p=\frac{P l^{2}}{E I}$ and $m=\frac{M l}{E I}$ are the normalized forces and moment applied at the tip point, $t=\frac{T}{l}$ is the dimensionless thickness, $\delta_{x}, \delta_{y}$ and $\delta_{\theta}$ are the normalized deflection parameters, respectively. It is obvious that the axial displacement $\Delta_{x}$ is comprised of a purely elastic component $x_{\mathrm{e}}$ resulting from the elastic stretch and a kinematic component $x_{\mathrm{k}}$ from the conservation of beam arc-length, as depicted in Fig. 1.

Accordingly, we can safely assume that the deformation of the beam can be divided into the following two steps:

1. The beam flexure stretches to $l+x_{\mathrm{e}}$ under the action of axial force.

2. The beam flexure with the length of $l+x_{\mathrm{e}}$ bends under the action of the generalized force.

\section{MPRB model for fixed-free beam flexures}

In this section, we consider a simple case for a fixed-free beam flexure with the length of $l$ subject to a combined force $[F, P]$, as shown in Fig. 2a. Based on the BCM model, the deformations of the free end $D$ can be easily derived by inserting $m=0$ into Eqs. (3)-(7).

Inspired by the PRB models proposed in Midha et al. (2015), Yu et al. (2016), a modified PRB model subject to a combined end force is proposed as depicted in Fig. 2b, which is composed of a rigid link $O D$ of length $\gamma l$ and an extension spring joined by a pin joint $O$ with a torsion spring. The undeflected length of the extension spring is $(1-\gamma) l$. We assume that the extension spring is not capable to bend to represent the axial stiffness, and the torsion spring represents the bending stiffness.

As illustrated in Fig. 2b, the extension spring is stretched by $x_{\mathrm{e}}$ with the axial force $P$, i.e., the rigid link $O D$ shifts to $O^{\prime} D^{\prime \prime}$. Thus the equivalent stiffness of the extension spring can be derived as

$$
\begin{aligned}
k_{\mathrm{e}} & =\frac{p}{\delta_{x \mathrm{e}}}=\frac{12}{t^{2}}, \\
K_{\mathrm{e}} & =\frac{k_{\mathrm{e}} E I}{l^{3}},
\end{aligned}
$$

where $k_{\mathrm{e}}$ is defined as the normalized stiffness of the extension spring.

Then the free end $D^{\prime \prime}$ rotates to $D^{\prime}$ around the pin joint $O^{\prime}$ under the combined end force. From Fig. 2b, we have the following equations:

$$
\begin{array}{ll}
\tan \frac{\Theta}{2} & =\frac{\delta_{x \mathrm{k}}}{\delta_{y}}, \\
\gamma \sin \frac{\Theta}{2} & =\frac{\sqrt{\delta_{x \mathrm{k}}^{2}+\delta_{y}^{2}}}{2},
\end{array}
$$

where $\Theta$ is denoted as the PRB angle and $\gamma$ is the characteristic radius factor.
It follows by Eqs. (3)-(7) and $m=0$ that

$$
\begin{aligned}
& \gamma=\frac{B_{f} f^{2}}{12600(5 p+12)^{2}(2 p+5)^{2}}+\frac{8750(5 p+12)^{2}}{B_{f}}, \\
& \Theta=\frac{f B_{f}}{5250(5 p+12)^{2}(2 p+5)},
\end{aligned}
$$

where

$$
\begin{aligned}
B_{f}= & 25(420-p)(5 p+12)^{2} \\
& +90(p-70)(2 p+5)(5 p+12) \\
& +36(2 p+5)^{2}(420-11 p) .
\end{aligned}
$$

The equivalent normalized torque $T_{f}$ applied at the torsion spring can be expressed as

$T_{f}=f \gamma \cos \Theta-p \gamma \sin \Theta \approx \gamma\left(f\left(1-\frac{\Theta^{2}}{2}\right)-p \Theta\right)$,

where $\sin \Theta$ and $\cos \Theta$ are approximated by $\Theta$ and $1-\frac{\Theta^{2}}{2}$ respectively because $\Theta$ is small enough.

Accordingly, the equivalent stiffness of the torsion spring can be calculated as

$$
\begin{aligned}
k_{\theta} & =\frac{T_{f}}{\Theta} \approx \gamma\left(\frac{5250(5 p+12)^{2}(2 p+5)}{B_{f}}-p\right), \\
K_{\Theta} & =\frac{k_{\theta} E I}{l},
\end{aligned}
$$

where $k_{\theta}$ is defined as the normalized stiffness of the torsion spring.

Meanwhile the location of the torsion spring (i.e., pin point $O$ ) can be derived as

$$
\begin{aligned}
x_{O} & =(1-\gamma) l, \\
x_{O^{\prime}} & =(1-\gamma) l+x_{\mathrm{e}}=\left(1-\gamma+\frac{t^{2} p}{12}\right) l .
\end{aligned}
$$

It is straightforward that the location of the torsion spring is not only determined by the characteristic radius factor $\gamma$, but also the axial force $P$. The PRB parameters (characteristic radius factor $\gamma$ and the equivalent torsional stiffness $K_{\Theta}$ ) are no longer constant values, but determined by the combined force. With this, the nonlinear characteristics of centershifting and load-stiffening are incorporated in the modified PRB model. Note that the MPRB model proposed in this paper can capture the nonlinear effect of the axial deformations and forces on the equivalent torsional stiffness with some simplifications compared with the BCM. More importantly, the proposed MPRB model inherits the advantage of the PRB models, which makes a wealth of existing rigidbody mechanism analysis and synthesis knowledge available to the treatment of compliant mechanisms and is convenient for the mechanism design, kinematic synthesis, as well as the structure parameter optimization. It is also worth pointing out that due to the limitation of the $\mathrm{BCM}$, the proposed 


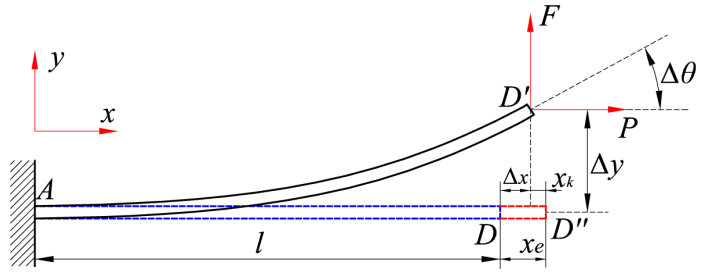

(a)

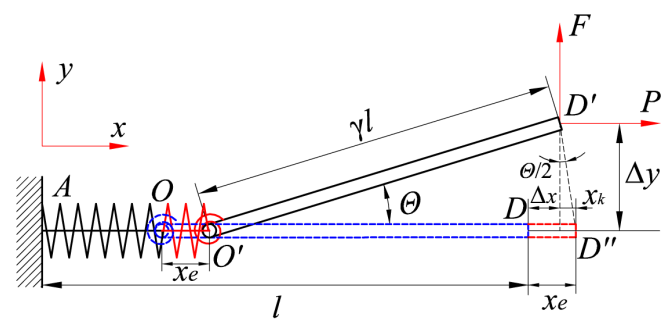

(b)

Figure 2. Fixed-free compliant beam. (a) Deflected configuration. (b) MPRB model.

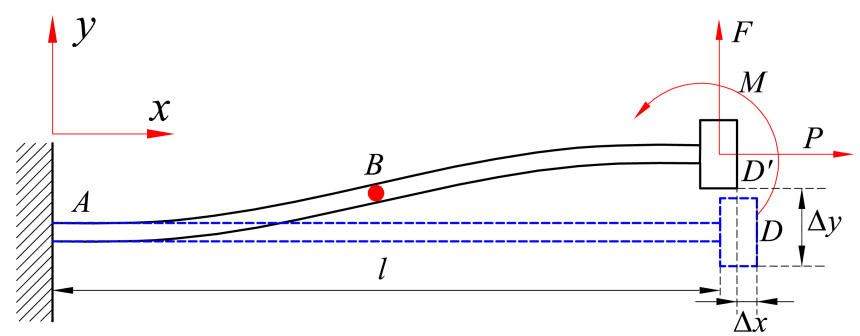

Figure 3. Deformation of the fixed-guided beam flexure with a single inflection point.

MPRB model can be only applied to the modeling of beam flexures with intermediate deflections where the transverse displacements are with an order of magnitude less than the beam length.

\section{MPRB model for fixed-guided beam flexures}

In this section, we consider the modeling of the fixed-guided beam flexures, as illustrated in Fig. 3. Because the slope of the beam is equivalent at the fixed and guided ends, there must be at least one point of inflection in the deformation curve. At every point of inflection, the internal moment vanishes. Without loss of generality, the initially-straight fixedguided beam with only one inflection point is considered in this work. Note that the methodology can be extended to the cases with more inflection points, which promises the capability of predicting the second mode bending of fixed-guided beams.

\subsection{Location of the inflection point}

As shown in Fig. 3, the fixed-guided beam flexure suffers from the generalized end force including lateral force $F$, axial force $P$ and moment $M$ such that the end section remains a constant angle in the process of deformation. According to Eqs. (1) and (2), we can obtain the curvature at the fixed end $A$ and guided end $D$ as

$$
\left.\frac{\mathrm{d} \theta}{\mathrm{d} s}\right|_{x=0}=M+F\left(l+\Delta_{x}\right)-P \Delta_{y}
$$

$$
\left.\frac{\mathrm{d} \theta}{\mathrm{d} s}\right|_{x=l+\Delta_{x}}=M .
$$

If the deformed beam has one inflection point $B$, the curvature at $A$ and $D$ must be opposite due to the same slope. Accordingly, we can derive the moment $M$ applied on the guided end as

$M=\frac{P \Delta_{y}-F\left(l+\Delta_{x}\right)}{2}$.

It is easy to verify that the equivalent moment applied at the midpoint of the deformation curve and the corresponding curvature equals to zero, which demonstrates that the inflection point is located at the mid-length of the fixed-guided beam.

\subsection{MPRB modeling of the fixed-guided beam}

An inflection point is characterized by a zero curvature and a zero moment, which allows it to be modeled as an instantaneous pin joint. Therefore, the fixed-guided beam flexure can be modeled as two fixed-free compliant segments with individual length of $l / 2$ joined at the midpoint $B$, as depicted in Fig. 4a. One of the two segments is fixed at the origin $A$ of the fixed-guided beam, and the other is fixed at the guided end $D$. Each segment can be simplified as a MPRB model with three dimensionless parameters $\gamma, k_{\mathrm{e}}$ and $k_{\theta}$. Accordingly, the fixed-guided beam flexure is modeled as a rigid link of length $\gamma l$ joined with two linear springs of stiffness $K_{\text {e } g}$ by two pin joints with two torsion springs of stiffness $K_{\theta g}$, as show in Fig. 4 b.

Similar to the fixed-free beams, we define the normalized parameters for the fixed-guided beam flexures as

$f=\frac{F l^{2}}{4 E I}, \quad p=\frac{P l^{2}}{4 E I}, \quad t=\frac{2 T}{l}$.

The characteristic radius factor $\gamma$ and the PRB angle $\Theta$ of the MPRB for fixed-guided beam flexures can be achieved by substituting the above load parameters into Eqs. (12) and (13). Then the equivalent stiffness can be calculated as

$K_{\mathrm{e} g}=\frac{k_{\mathrm{e}} E I}{\left(\frac{l}{2}\right)^{3}}=\frac{96 E I}{l^{3} t^{2}}$, 


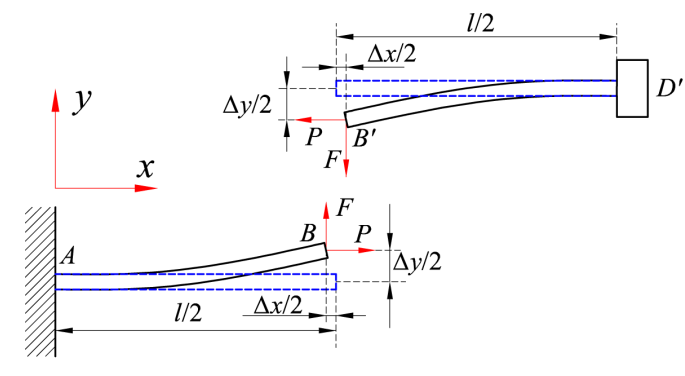

(a)

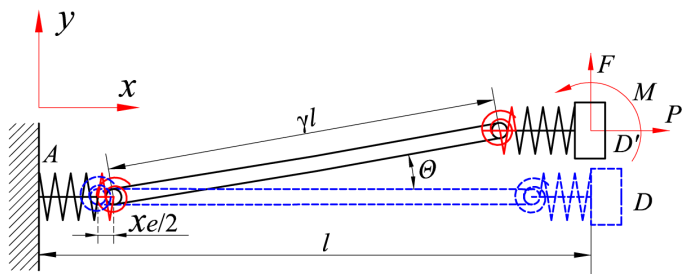

(b)

Figure 4. Fixed-guided beam. (a) Two fixed-free compliant segments. (b) MPRB model.

Table 1. Geometric parameters of the fixed-guided beam flexure.

\begin{tabular}{lrrr}
\hline Parameters & $l(\mathrm{~mm})$ & $b(\mathrm{~mm})$ & $T(\mathrm{~mm})$ \\
\hline Value & 30.0 & 10.0 & 0.5 \\
\hline
\end{tabular}

$$
\begin{aligned}
K_{\theta g} & =\frac{k_{\theta} E I}{\frac{l}{2}} \\
& =\frac{2 \gamma E I\left(5250(5 p+12)^{2}(2 p+5)-p B_{f}\right)}{l B_{f}} .
\end{aligned}
$$

Hence the displacement of the guided end $D$ can be derived as

$$
\begin{aligned}
& \Delta_{y}=\gamma l \sin \Theta=\gamma l \Theta=\frac{\gamma l B_{f} f}{5250(5 p+12)^{2}(2 p+5)} \\
& \Delta_{x}=2\left(\frac{P}{K_{\mathrm{e} g}}-\frac{\gamma l}{2}(1-\cos \Theta)\right)=l\left(\frac{p t^{2}}{12}-\frac{\gamma \Theta^{2}}{2}\right)
\end{aligned}
$$

It is clear that the proposed MPRB model is capable of capturing the load-dependent property of the fixed-guided beam flexures. Compared with the recent developed Bi-BCM model for fixed-guided beams (Ma and Chen, 2017), the proposed MPRB model demonstrates a similar change trend and prediction accuracy, as demonstrated in Fig. 5, where the key structure parameters of the fixed-guided beam are listed in Table 1 . The small discrepancy is caused by some simplifications in the MPRB model. With the proposed MPRB model, the traditional kinematic and dynamic analysis methods for rigid-body mechanisms can be extended to the synthesis of the fixed-guided beams based compliant mechanisms.

\subsection{Model verification with FEA}

In this section the developed MPRB modeling method for fixed-guided beam flexures is verified by the FEA software ANSYS. According to the structure illustrated in Fig. 3, the finite element model of fixed-guided beam flexure model is established with the key structure parameters listed in Table 1 , where $b$ is the width of the beam flexure. The aluminum alloy A17075-T6 with Young's modulus of $71 \mathrm{GPa}$ is
Table 2. The maximum error for the tip point ( $\mathrm{mm})$.

\begin{tabular}{lrrrr}
\hline Cases & MPRB & PRB 1R & 3-Spring & PRR \\
\hline$F=5 \mathrm{~N}$ & 0.02 & 0.17 & 0.07 & 0.06 \\
$P=0 \mathrm{~N}$ & 0.04 & 0.12 & 0.08 & 0.07 \\
$P=20 \mathrm{~N}$ & 0.02 & 0.17 & 0.14 & 0.13 \\
$P=-20 \mathrm{~N}$ & 0.11 & 0.24 & 0.17 & 0.13 \\
\hline
\end{tabular}

adopted as the material. In addition, one end of the beam is fixed and the slope angle of the other end is constrained to zero.

A constant transverse force $F=5 \mathrm{~N}$ with axial forces $P$ ranging from -40 to $40 \mathrm{~N}$ are applied at the guided end. As demonstrated in Fig. 6c, the equivalent torsional stiffness of the beam flexure increases with the increase of the axial force due to the load-stiffening effect. Compared with the FEA results, the $y$-axial deflection error of the proposed MPRB modeling method is less than $1.1 \%$ and the maximum error for the tip point is about $0.1 \%$ of the beam length as shown in Fig. 6b.

It is also interesting to compare the proposed MPRB model with the existing PRB models. In particular, the PRB 1R model (Midha et al., 2015), PRB 3-Spring model (Venkiteswaran and $\mathrm{Su}, 2016$ ) and PRB PRR model (Yu et al., 2016) are adopted to model each fixed-free segment. The corresponding results are plotted in Fig. 6. It is obvious that the maximum error for the tip point obtained by the MPRB method is improved by $88.2,71.4$ and $66.7 \%$ compared with PRB 1R, 3-Spring and PRR modeling methods.

Moreover, Fig. 7 shows the simulated (FEA) and approximated (MPRB, PRB 1R, 3-Spring and PRR) tip loci for three cases: $P=0, P=-20 \mathrm{~N}$ and $P=20 \mathrm{~N}$, respectively. As listed in Table 2, the errors between the proposed MPRB model and the FEA analysis are less than $0.4 \%$ of beam length in the presence of axial compression and $0.1 \%$ in the presence of axial tension. Compared with PRB 1R, PRB $3-$ Spring and PRB PRR modeling methods, the modeling precision of the proposed MPRB is improved by 54.2, 35.2 and 


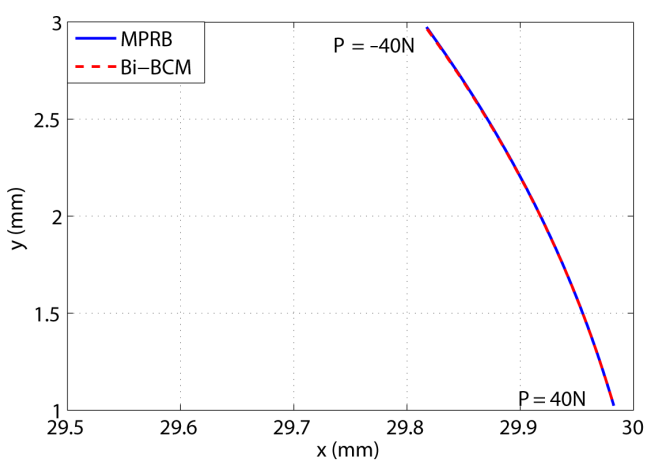

(a)

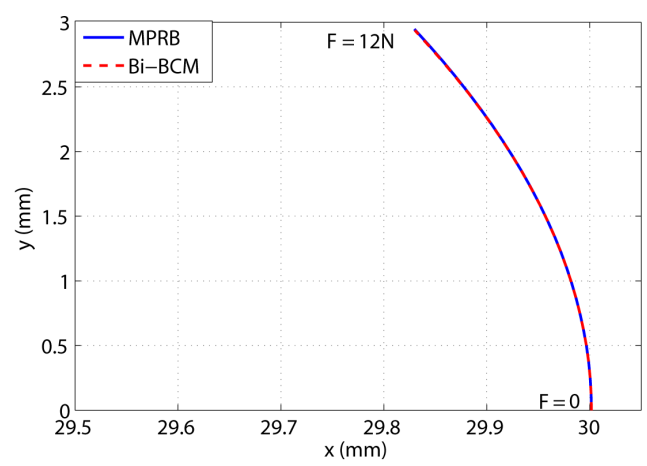

(b)

Figure 5. Comparisons between the proposed MPRB model and Bi-BCM model. (a) $F=5 \mathrm{~N}$ with different axial forces. (b) $P=20 \mathrm{~N}$ with different transverse forces.

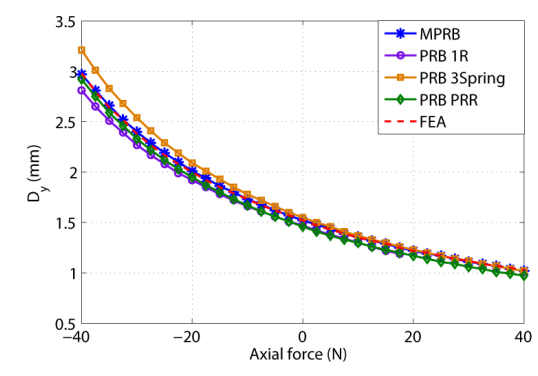

(a)

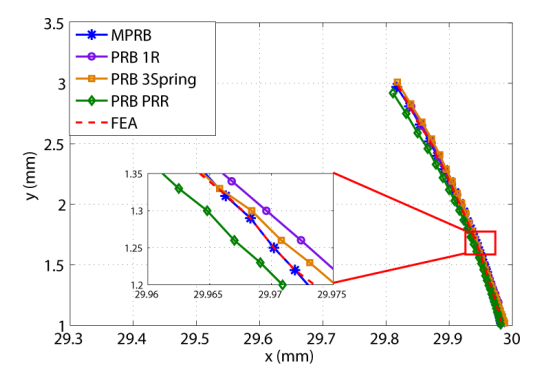

(b)

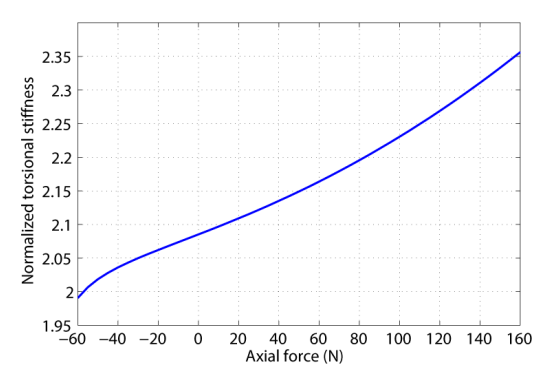

(c)

Figure 6. The deflection loci of the fixed-guided beam flexure with $F=5 \mathrm{~N}$. (a) $Y$-axial deflection. (b) Deflection loci of the guided end. (c) Normalized torsional stiffness.

$15.4 \%$ for axial compression and 88.2, 85.7 and $84.6 \%$ for axial tension, respectively.

\section{Case studies}

In this section, the proposed MPRB model is further applied to the analysis of three compliant mechanisms with fixedguided beams, including a compound parallelogram mechanism, a bistable mechanism and a 1-DOF translational mechanism, where the impacts of the axial deformations and the axial forces cannot be ignored.

\subsection{Compound compliant parallelogram mechanism}

As depicted in Fig. 8a, the compound compliant parallelogram mechanism consists of two parallelogram mechanisms connected in parallel. With the action of the driving force $F_{y}$, each beam deflects in an "S"-shaped form. Based on the proposed modeling approach, we establish the MPRB model of the compound compliant parallelogram mechanism, as demonstrated in Fig. 8b.

We here define $F_{\mathrm{b}}$ and $P_{\mathrm{b}}$ as the lateral force and axial force applied at each beam respectively. According to the equilibrium condition of forces, we can achieve the following relationship as

$$
F_{\mathrm{b}}=\frac{F_{y}}{4}, \quad f_{\mathrm{b}}=\frac{F_{\mathrm{b}} l^{2}}{4 E I}, \quad p_{\mathrm{b}}=\frac{P_{\mathrm{b}} l^{2}}{4 E I},
$$

where $f_{\mathrm{b}}$ and $p_{\mathrm{b}}$ are the normalized lateral and axial force, respectively.

According to the modeling process for the fixed-guided beams, we can derive the output displacement $\Delta_{y}$ and the equivalent stiffness $K_{y}$ in the direction of motion ( $y$-axis) of the compound compliant parallelogram mechanism as

$$
\begin{aligned}
& \Delta_{y}=\gamma l \Theta=\frac{\gamma l B_{f} f_{\mathrm{b}}}{5250\left(5 p_{\mathrm{b}}+12\right)^{2}\left(2 p_{\mathrm{b}}+5\right)} . \\
& K_{y}=\frac{F_{y}}{\Delta_{y}}=\frac{84000 E I\left(5 p_{\mathrm{b}}+12\right)^{2}\left(2 p_{\mathrm{b}}+5\right)}{\gamma l^{3} B_{f}},
\end{aligned}
$$

where the corresponding axial force $\left(P_{\mathrm{b}}\right.$ and $\left.p_{\mathrm{b}}\right)$ can be further calculated by letting Eq. (25) to 0 since the horizontal length remains unchanged.

Based on the above results, the deflection-force curve can be obtained as depicted in Fig. 9, which shows a strong nonlinearity of the equivalent stiffness, which agrees well with FEA results with a discrepancy less than $2.1 \%$. To be specific, the equivalent stiffness increases with the output displacement. Meanwhile the changing rate of the equivalent 


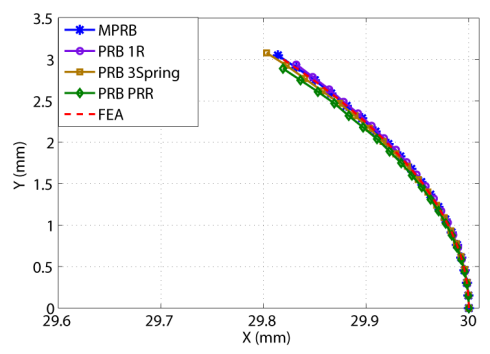

(a)

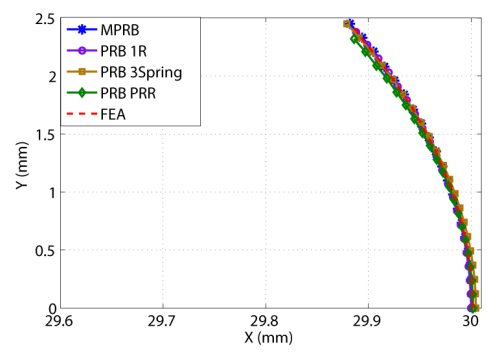

(b)

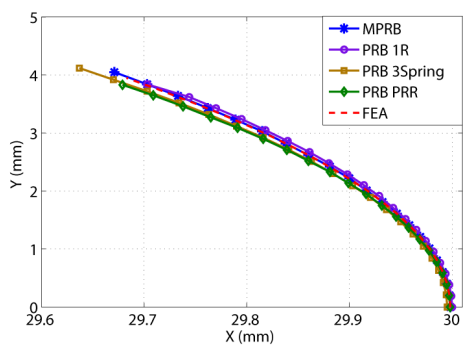

(c)

Figure 7. The deflection loci of the fixed-guided beam flexure. (a) $P=0$. (b) $P=20$ N. (c) $P=-20$ N.

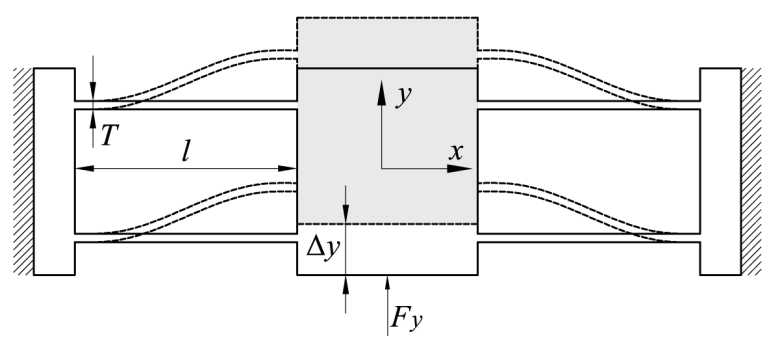

(a)

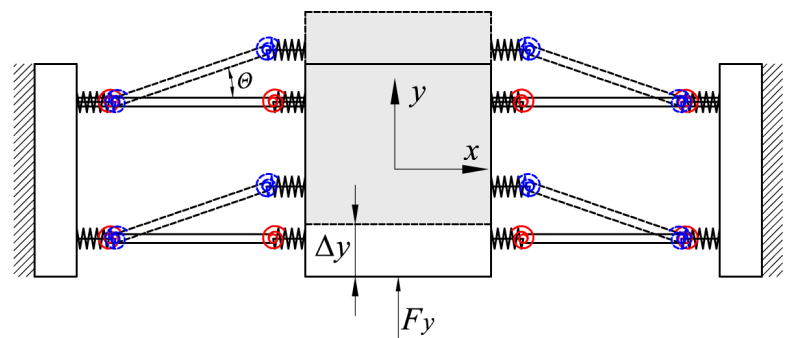

(b)

Figure 8. (a) Compound compliant parallelogram mechanism. (b) MPRB model

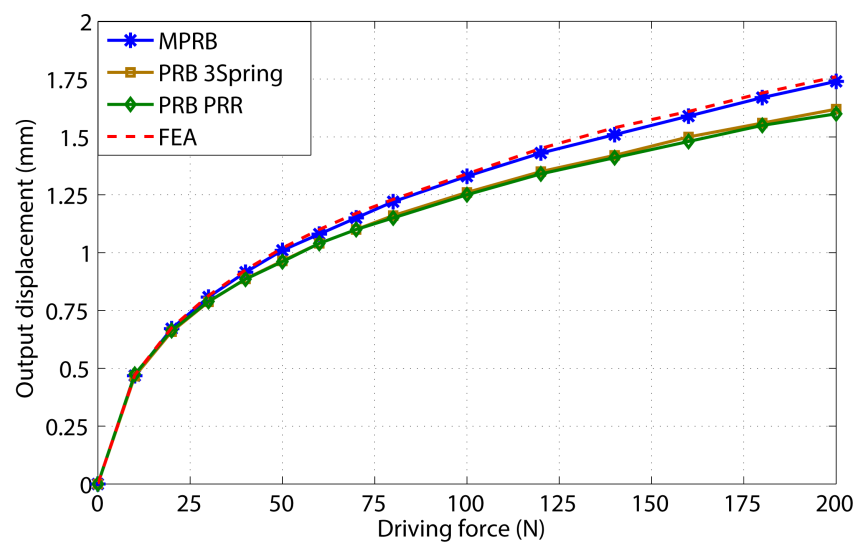

Figure 9. Output displacements of the compound compliant parallelogram mechanism.

stiffness also increases with the output displacement. For comparison purposes, the results obtained by PRB 3-Spring and PRR models are also plotted in Fig. 9, which demonstrates a modeling error of 7.9 and $9.1 \%$ respectively. The reason behind the discrepancies (of the existing methods) is that the axial tensile force applied to each beam flexure increases with the output displacement and significantly changes the stiffness of the beam flexures.

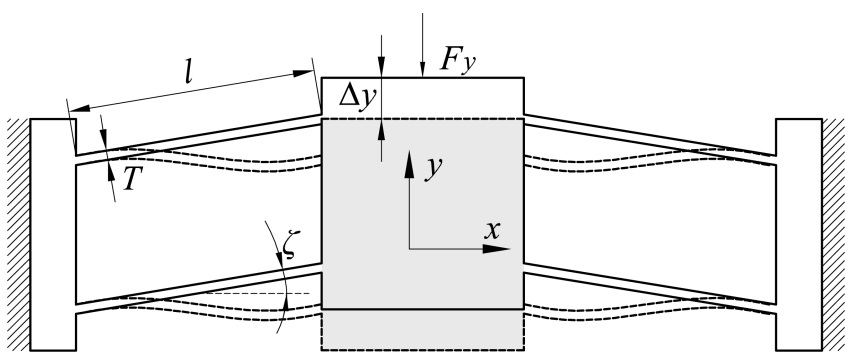

Figure 10. Fixed-guided bistable compliant mechanism.

\subsection{Bistable mechanism}

Another commonly investigated compliant mechanism with fixed-guided beam flexures is the compliant bistable mechanism. Figure 10 shows a typical bistable compliant mechanism consisting of four identical fixed-guided straight beams, where the impact of axial force cannot be ignored in case of deformed configurations. A fixed-guided beam taken from the bistable compliant mechanism is plotted in Fig. 11a. The geometric parameters of the beam are listed in Table 3 . Again, Al7075-T6 is adopted as the material.

We introduce the coordinate frame such that $X$-axis is along the beam with the origin at the fixed end, as shown in Fig. 11a. For a given vertical displacement $\Delta_{y}$ at the guided end, the corresponding deflection parameters in the $X Y$ coordinate frame can be normalized as

$\delta_{Y}=\frac{2 \Delta_{Y}}{l}=\frac{2 \Delta_{y} \cos \zeta}{l}$, 


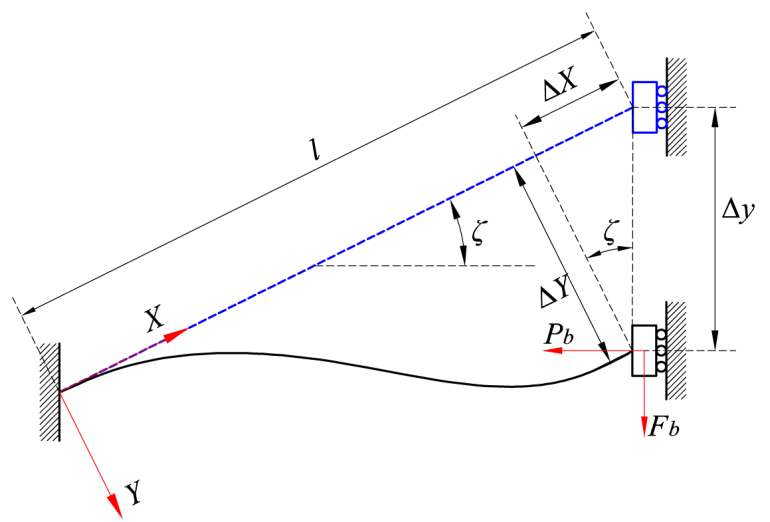

(a)

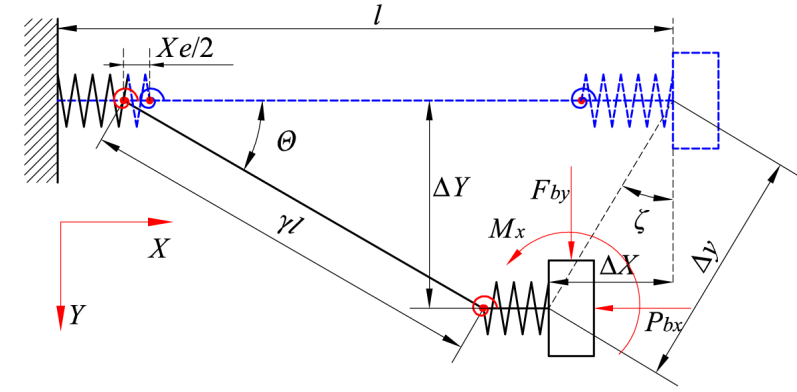

(b)

Figure 11. A fixed-guided beam in the bistable mechanism. (a) Schematic diagram. (b) MPRB model.

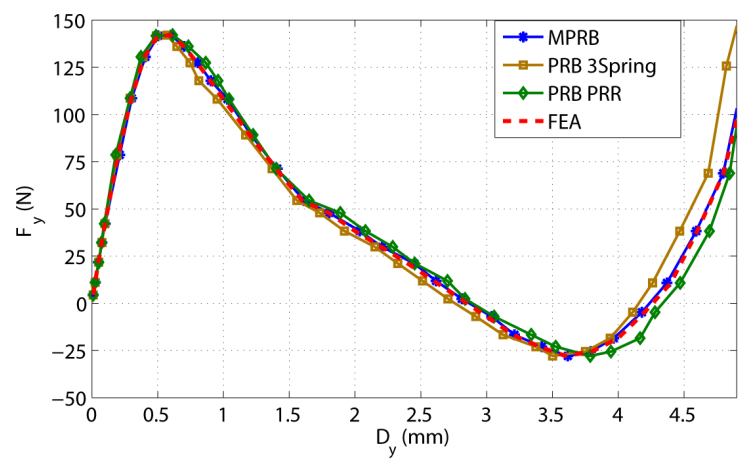

(a)

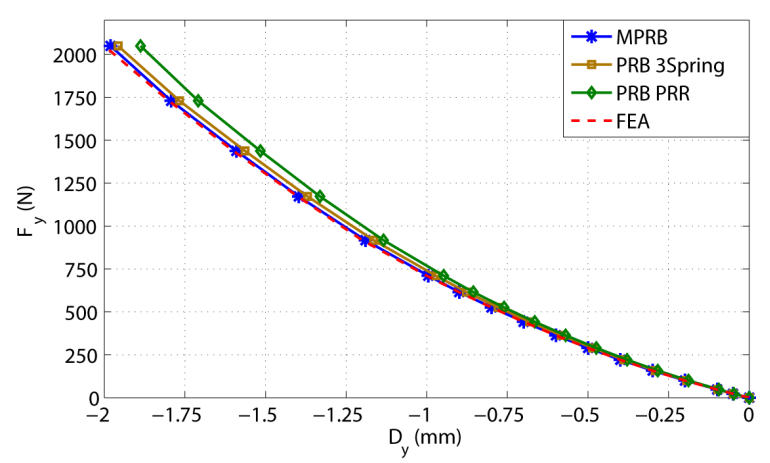

(b)

Figure 12. The force-deflection curves of the bistable mechanism. (a) Positive displacement. (b) Negative displacement.

Table 3. Key geometric parameters of the bistable compliant mechanism.

\begin{tabular}{lrrrr}
\hline Parameters & $l(\mathrm{~mm})$ & $b(\mathrm{~mm})$ & $T(\mathrm{~mm})$ & $\zeta\left(^{\circ}\right)$ \\
\hline Value & 30.0 & 10.0 & 0.5 & 5.5 \\
\hline
\end{tabular}

$\delta_{X}=\frac{2 \Delta_{X}}{l}=-\frac{2 \Delta_{y} \sin \zeta}{l}$

where $\zeta$ represents the angle between the horizontal line and the undeformed beam configuration.

According to the proposed modeling method, we can establish the PRMB model for the fixed-guided beam of the bistable mechanism, as depicted in Fig. $11 \mathrm{~b}$, where $F_{b y}$ and $F_{b x}$ represent the lateral force and axial force applied at the guided end of the beam flexure respectively. According to the equilibrium condition of forces, we can achieve the normalized load parameters as

$f_{\mathrm{b} y}=\frac{F_{\mathrm{b} y} l^{2}}{4 E I}=\frac{\left(F_{\mathrm{b}} \cos \zeta-P_{\mathrm{b}} \sin \zeta\right) l^{2}}{4 E I}$ $p_{\mathrm{b} x}=\frac{P_{\mathrm{b} x} l^{2}}{4 E I}=-\frac{\left(P_{\mathrm{b}} \cos \zeta+F_{\mathrm{b}} \sin \zeta\right) l^{2}}{4 E I}$,

where $F_{\mathrm{b}}$ and $P_{\mathrm{b}}$ are the vertical and horizontal forces applied at each beam flexure shown in Fig. 11a.

Similar to the modeling procedure of the compound compliant parallelogram mechanism, we can substitute $f=f_{\mathrm{b} y}$, $p=p_{\mathrm{b} x}$ and the dimensionless thickness $t=\frac{2 T}{l}$ in Eqs. (24) and (25), and derive the load-deflection curve for the bistable mechanism.

We first let the input displacement $\delta_{y}$ increase from 0 to $5 \mathrm{~mm}$. The resulting load-deflection curve is plotted in Fig. 12a. Compared with the FEA results, the proposed MPRB model can efficiently describe the nonlinear relationship between the driving force and the output displacement of the bistable mechanisms with an error less than $4.2 \%$. The existing methods such as PRB 3-Spring and PRR models considering the effect of axial deformations shows an modeling error of 9.3 and $9.6 \%$ respectively. It is worth pointing out that there are two stable equilibrium positions and an unstable equilibrium position of the bistable mechanism shown in Fig. 12a. The proposed MPRB method works well at both stable and unstable equilibrium positions. 


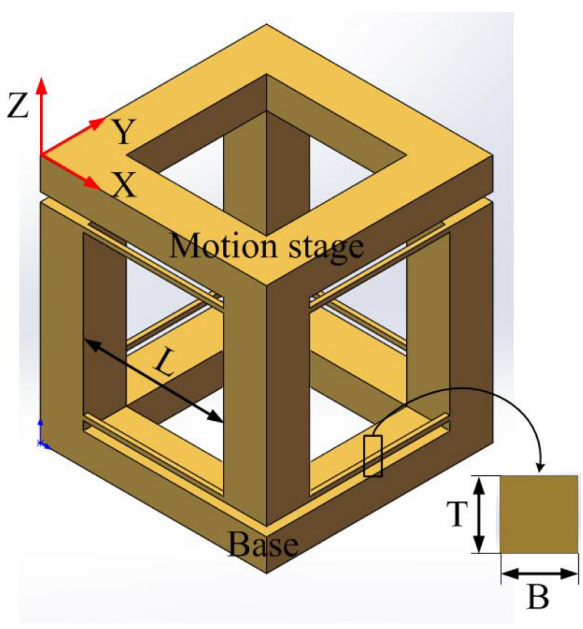

(a)

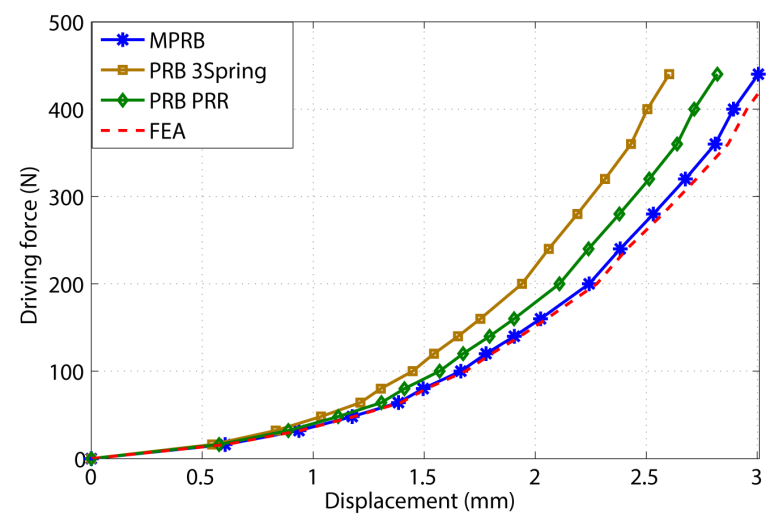

(b)

Figure 13. (a) 8-beam flexures based 1-DOF translational mechanism. (b) The force-deflection curves.

On the other hand, we apply negative displacement ranging from 0 to $-2 \mathrm{~mm}$ to the bistable mechanism. As illustrated in Fig. 12b, the equivalent stiffness significantly increases with the increase of the displacement. Compared with FEA results, the proposed MPRB demonstrates a modeling error less than $1.1 \%$, while the maximum modeling errors of the PRB 3-Spring and PRR models are 3.0 and $6.2 \%$ respectively. The comparison results indicate the effectiveness and accuracy of the proposed MPRB model to predict the nonlinear behavior and the unstable equilibrium positions of the bistable mechanism.

\subsection{1-DOF translational mechanism}

As depicted in Fig. 13a, a symmetric and compact 1-DOF mechanism presented by Hao (2016) consists of 8 fixedguided wire beams. Under the constraint of the wire beams, the motion stage can achieve precise translation along $z$ axis. In this section, the finite element model of the 1-DOF mechanism is established with these key structure parameters: $L=30(\mathrm{~mm}), T=B=1(\mathrm{~mm})$. Al7075-T6 is also selected as the material.

Note that each wire beam of the 1-DOF translational mechanism deflects in an "S"-shaped form where the horizontal length remains unchanged. Through the similar modeling process for the compound compliant parallelogram mechanism, we can establish the MPRB model for the 1DOF mechanism. The corresponding force-deflection curve can be derived as plotted in Fig. 13b, which agrees well with the FEA results with an error less than $2.2 \%$. The results also indicate a significant nonlinear load-stiffening effect during the deformations, which should be considered during the modeling process. Compared with the existing PRB 3-Spring and PRR models, the modeling precision is improved by 85.5 and $73.2 \%$ respectively.

\section{Conclusions}

In this paper, we took the nonlinear effect (center-shifting and load-stiffening) caused by axial deformations and forces into consideration and developed a modified pseudo-rigidbody modeling method for compliant mechanisms with fixed-guided beam flexures. Based on the BCM, the fixedfree beam flexure was modeled as a rigid link and an extension spring joined by a pin joints with a torsion spring, where the PRB parameters including the characteristic radius factor and the equivalent torsional stiffness were shown to be determined by the general tip loads, instead of a constant value. Accordingly, a fixed-guided beam flexure was modeled as a pair of two fixed-free beam flexures jointed at the inflection point, where each fixed-free beam flexure was modeled by the proposed MPRB method. FEA simulations and three case studies, including a compound parallelogram mechanism, a fully compliant bistable mechanism and a 1-DOF translational mechanism, demonstrated significant improvement over existing results to predict performance characteristics of the compliant mechanisms with fixed-guided beams. It is worth pointing out that the proposed MPRB can be extended to the cases with more inflection points, which promises the capability of predicting the second mode bending of fixedguided beams. Also note that the proposed MPRB modeling method is only suitable for capturing the deflection behavior of fixed-guided beam flexures with known inflection points and intermediate deformations, due to the limitation of the BCM. Future extensions along this line of research include the pseudo-rigid-body modeling for the case of larger deformations and more generalized flexure hinges (both notched hinges and beam flexures) by considering the influence of the axial force. 
Data availability. All the data used in this manuscript can be obtained by requesting from the corresponding author.

Competing interests. The authors declare that they have no conflict of interest.

Acknowledgements. We would like to thank the financial support from the NSFC under Grant no. 61327003, the National Key Research and Development Program of China under Grant no. 2017YFF0105903, and the Fundamental Research Funds of Shandong University under Grant no. 2015JC034.

Edited by: Chin-Hsing Kuo

Reviewed by: two anonymous referees

\section{References}

Awtar, S., Slocum, A. H., and Sevincer, E.: Characteristics of beambased flexure modules, J. Mech. Des.-T. ASME, 129, 625-639, 2007.

Chen, G. and Ma, F.: Kinetostatic modeling of fully compliant bistable mechanisms using Timoshenko beam constraint model, J. Mech. Des., 137, 022301, https://doi.org/10.1115/1.4029024, 2015.

Ding, B., Li, Y., Xiao, X., Tang, Y., and Li, B.: Design and analysis of a 3-DOF planar micromanipulation stage with large rotational displacement for micromanipulation system, Mech. Sci., 8, 117126, https://doi.org/10.5194/ms-8-117-2017, 2017.

Hao, G.: Determine design and analytical analysis of a class of symmetrical flexure guiding mechanisms for linear actuators, J. Mech. Des.-T. ASME, 139, 012301, https://doi.org/10.1115/1.4034579, 2017.

Hao, G. and Li, H.: Extended static modelling and analysis of compliant compound parallelogram mechanisms considering the initial internal axial force, J. Mech. Robot., 8, 041008, https://doi.org/10.1115/1.4032592, 2016.

Howell, L. L.: Compliant mechanisms, John Wiley \& Sons, New York, 2001.
Liu, P. and Yan, P.: A new model analysis approach for bridge-type amplifiers supporting nano-stage design, Mech. Mach. Theory, 99, 176-188, 2016.

Liu, P., Yan, P., Zhang, Z., and Leng, T.: Modeling and control of a novel X-Y parallel piezoelectric-actuator driven nanopositioner, ISA Trans., 56, 145-154, 2015.

Luo, Y. and Liu, W.: Analysis of the displacement of distributed compliant parallel-guiding mechanism considering parasitic rotation and deflection on the guiding plate, Mech. Mach. Theory, 80, 151-165, 2014.

Ma, F. and Chen, G.: Bi-BCM: A Closed-Form Solution for FixedGuided Beams in Compliant Mechanisms, J. Mech. Robot., 9, 014501, https://doi.org/10.1115/1.4035084, 2017.

Midha, A., Bapat, S. G., Mavanthoor, A., and Vivekananda, C.: Analysis of a fixed-guided compliant beam with an inflection point using the pseudo-rigid-body model concept, J. Mech. Robot., 7, 031007, https://doi.org/10.1115/1.4028131, 2015.

Parmar, G., Barton, K., and Awtar, S.: Large dynamic range nanopositioning using iterative learning control, Precis. Eng., 38, 48-56, 2014.

$\mathrm{Su}, \mathrm{H}$. J.: A pseudorigid-body 3R model for determining large deflection of cantilever beams subject to tip loads, J. Mech. Robot., 1, 021008, https://doi.org/10.1115/1.3046148, 2009.

Teo, T. J., Chen, I. M., Yang, G., and Lin, W.: A generic approximation model for analyzing large nonlinear deflection of beambased flexure joints, Precis. Eng., 34, 607-618, 2010.

Turkkan, O. A. and Su, H.-J.: DAS-2D: a concept design tool for compliant mechanisms, Mech. Sci., 7, 135-148, https://doi.org/10.5194/ms-7-135-2016, 2016.

Venkiteswaran, V. K. and Su, H. J.: A 3-Spring pseudorigid-body model for soft joints with significant elongation effects, J. Mech. Robot., 8, 061001, https://doi.org/10.1115/1.4032862, 2016.

Yu, Y. Q., Feng, Z. L., and Xu, Q. P.: A pseudo-rigid-body 2R model of flexural beam in compliant mechanisms, Mech. Mach. Theory, 55, 18-33, 2012.

Yu, Y. Q., Zhou, P., and Xu, Q. P.: A new pseudo-rigid-body model of compliant mechanisms considering axial deflection of flexural beams, New Trends in Mechanism and Machine Science, Springer International Publishing, 851-858, 2015.

Yu, Y. Q., Zhu, S. K., Xu, Q. P., and Zhou, P.: A novel model of large deflection beams with combined end loads in compliant mechanisms, Precis. Eng., 43, 395-405, 2016 